\title{
Bayesian active learning for electromagnetic structure design
}

\author{
Jixiang Qing*, Nicolas Knudde, Ivo Couckuyt, Domenico Spina, Tom Dhaene \\ IDLab, Department of Information Technology, Ghent University-imec, \\ Technologiepark-Zwijnaarde 126, 9052 Ghent, Belgium \\ *Jixiang.Qing@UGent.be
}

\begin{abstract}
A novel design framework based on Bayesian active learning is presented in this contribution. The proposed approach allows one to identify a set of design configurations satisfying the chosen specification. In particular, the entropy search-based active learning strategy, which relies on a Gaussian Process model, is able to minimize the number of time-consuming computer simulations or expensive design trials necessary to reach this goal. A suitable application example validates the proposed method.
\end{abstract}

Index Terms-active learning, Gaussian Process, target region

\section{INTRODUCTION}

The design of modern electromagnetic and electronic circuits is a complex task. Indeed, microwave designers have to make the proper tradeoffs between conflicting design requirements considering physical effects such as reflection, crosstalk and propagation delays. In this scenario, Computer-Aided Design (CAD) simulations are an essential tool. However, due to the increased bandwidth and complexity of modern electromagnetic and electronic circuits, simulations are often expensive, both in terms of computational time and resources. Hence, it is of paramount importance to limit the number of (expensive) simulations during the design phase.

In order to tackle this problem, several macromodeling techniques have been developed in the last decade [1]-[3]. The main goal of these approaches is to compute via a limited number of expensive simulations (i.e. full-wave electromagnetic simulations) a mathematical model of the system under study describing the system behavior as seen from its $\mathrm{I} / \mathrm{O}$ ports, with respect to a set of design parameters (such as the width or length of a metallic trace). Once a macromodel is computed, it can efficiently replace physics-based simulators for different design activities, such as design space exploration, optimization and sensitivity analysis [1]-[3]. These approaches rely on the assumption that is possible to compute an accurate macromodel in the entire range of variation of the design parameters (also called design space) via a limited number of CAD simulations. However, this condition does not hold when the system considered has a very dynamic behavior and/or the number of design parameters is high (curse of dimensionality).

In this contribution, a different methodology is proposed: the main idea is to directly identify the areas of the design space which satisfy a suitable objective chosen by the designers (for example the value of the cut-off frequency or

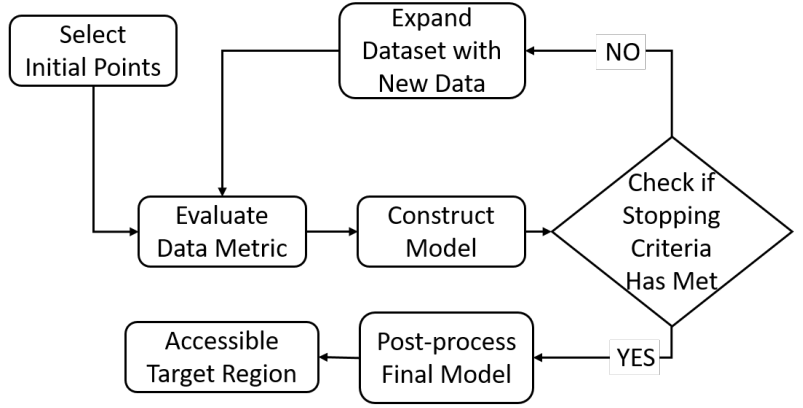

Fig. 1: Flowchart of the active learning algorithm.

the bandwidth of a filter): these areas are defined as target or feasible regions in the rest of the paper. In particular, we introduce an information-theoretic active learning technique based on Gaussian Process (GP) modeling, which is able to sequentially find the regions of interest in the design space. Since simulations are computationally expensive, our goal is to get as much information as possible about the target region with as little evaluations as possible, which falls exactly within the scope of active learning. Finally, in order to clearly represent the target region with respect to the value of the design parameters, its boundaries are expressed via a bounding hypercube based on Principle Component Analysis (PCA) [4].

The remainder of this paper is organized as follows. The proposed active learning-based modeling framework is described in Section II, while a suitable application example is presented in Section III. Finally, conclusions are drawn in Section IV.

\section{Methodology}

\section{A. Active Learning for target region discovery}

Active learning is also known as the sequential experimental design method in statistics [5]: it intelligently and sequentially extends the dataset to reveal the desired properties of the model [6]. Such desired properties can either be the maximum location of a function [7], [8], a set of differential equations [5], or a target region of interest, which is the case considered in our study.

The basic workflow of active learning is illustrated in Fig. 1: an initial set of design parameters values is selected via design of experiments (DOE) techniques. Subsequently, the 
corresponding function values are evaluated. A regression model is then constructed to fit this data. Next, a new sample of the design parameters is selected according to some specified criterion built upon the model (sampling strategy). The main difference with respect to many macromodeling techniques is that the model employed here is not deterministic, but stochastic, as described in Section II-B. Hence, the sampling strategy employs the model prediction on the location of the target region to choose the new sample to evaluate. Once a new simulation is performed, the model is updated accordingly. This process is repeated until a stopping criterion is met. Finally, the location of the target region can be estimated from the obtained model using a post-processing step. In the following, the proposed method is described assuming that a single target region can be identified in the design space, for simplicity. However, the proposed method can be applied also when disjoint feasible regions are present.

\section{B. Gaussian Process modeling}

There are many supervised machine learning models that can be utilized to perform regression, such as Neural Networks, support vector regression (SVR) and GPs. Nevertheless, under the category of data efficient machine learning, GP modeling is arguably the most prevalent technique. GPs have been widely used as a regression method in many different areas including electronic engineering [9], computational fluid dynamics [10] and additive manufacturing [11].

A GP can be considered as a distribution over functions $f: \mathcal{X} \rightarrow \mathbb{R}$, that is completely defined by a suitable mean function $m: \mathcal{X} \rightarrow \mathbb{R}$ and covariance function $k: \mathcal{X} \times \mathcal{X} \rightarrow \mathbb{R}$, where the type of mean and covariance functions depends on the particular problem under study. Now, starting from the GP and the training data $D_{n}$, it is possible to derive a belief of the function distribution (called posterior in the GP framework). Such belief is Gaussian with mean and variance defined as:

$$
\begin{gathered}
\mu\left(\boldsymbol{x}_{*}\right)=\mathbb{E}\left(f_{*} \mid \boldsymbol{x}_{*}, D_{n}\right)=k_{* n}^{T}\left(K_{n n}+\sigma^{2} I\right)^{-1} y \\
\sigma^{2}\left(\boldsymbol{x}_{*}\right)=\mathbb{V}\left[f_{*} \mid \boldsymbol{x}_{*}, D_{n}\right]=K_{* *}-K_{n *}^{T}\left(K_{n n}+\sigma^{2} I\right)^{-1} K_{n *}
\end{gathered}
$$

where $\boldsymbol{x}_{*}$ denotes the test point, $K_{n n}$ is the kernel matrix between training samples $D_{n}, K_{n *}$ denotes the kernel matrix between the training samples and the test point, $\sigma^{2}$ is the variance of Gaussian likelihood, which usually represents the noise level of training sample. In this paper, the mean function $m$ is chosen equal to zero, which is a common assumption [6], [12], [13], while the Automatic Relevance Detection [13] (ARD) version of the Squared Exponential (SE) kernel (also known Radial Basis Function (RBF)) is employed as covariance function:

$$
k\left(\boldsymbol{x}, \boldsymbol{x}^{\prime}\right)=\sigma_{k}^{2} \exp \left(-\sum_{d=1}^{D} \frac{\boldsymbol{x}_{d}-\boldsymbol{x}_{d}^{\prime 2}}{2 \ell_{d}}\right)
$$

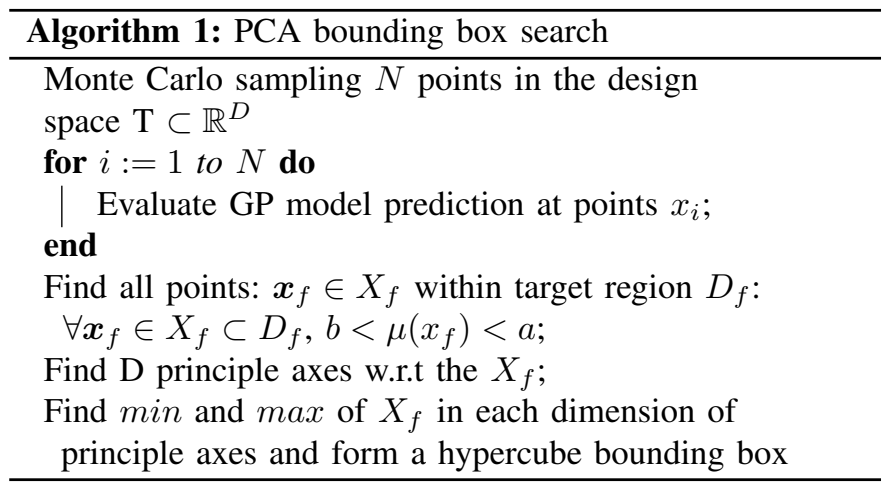

The hyperparameters $\theta=\left\{\ell_{d}, \sigma_{k}, \sigma\right\}$ are determined using the Maximum Likelihood Estimate (MLE):

$$
\begin{aligned}
& \theta_{M L E}=\underset{\theta}{\operatorname{argmax}} \log p(\boldsymbol{y} \mid X, \boldsymbol{\theta})= \\
& \underset{\theta}{\operatorname{argmax}}\left(-\frac{1}{2} \boldsymbol{y}^{T} K_{n n}{ }^{-1} \boldsymbol{y}-\frac{1}{2} \log \left|K_{n n}\right|-\frac{n}{2} \log (2 \pi)\right)
\end{aligned}
$$

The interested reader is referred to [13] for a detailed description of the properties of GPs.

\section{Entropy Search-based Sequential Sampling}

The sampling strategy can be defined by an acquisition function. In this paper, the acquisition function presented in [6] is adopted. The main intuition of the approach described in [6] is to sample the point that maximizes the information gain of a target region $g$, which is quantified by differential entropy:

$$
\alpha(\boldsymbol{x})=\mathbb{H}\left(p\left(g \mid D_{n}\right)\right)-\mathbb{E}_{p\left(f_{\boldsymbol{x}} \mid D_{n}, \boldsymbol{x}\right)}\left[\mathbb{H}\left(p\left(g \mid D_{n} \cup\left\{\boldsymbol{x}, f_{\boldsymbol{x}}\right\}\right)\right)\right]
$$

where $p\left(f_{\boldsymbol{x}} \mid D_{n}, \boldsymbol{x}\right)$ denotes the predictive distribution at $\boldsymbol{x}$.

Let us suppose that, in the problem under study we are interested in the target region defined as: $b<f<a$. Hence, the goal is to maximize the information gain about the region of interest and its complement. This can be rewritten using properties of mutual information [14], and the acquisition function becomes:

$$
\begin{aligned}
\alpha_{g}(\boldsymbol{x})= & 3 \mathbb{H}\left(p\left(f \mid D_{n}, \boldsymbol{x}\right)\right)-\mathbb{H}\left(p\left(f \mid D_{n}, \boldsymbol{x}, f>b\right)\right) \\
& -\mathbb{H}\left(p\left(f \mid D_{n}, \boldsymbol{x}, b<f<a\right)\right) \\
& -\mathbb{H}\left(p\left(f \mid D_{n}, \boldsymbol{x}, a<f\right)\right)
\end{aligned}
$$

In particular, the following closed form expression holds [6]:

$$
\begin{aligned}
& \mathbb{H}\left(p\left(f \mid D_{n}, \boldsymbol{x}\right)\right)-\mathbb{H}\left(p\left(f \mid D_{n}, \boldsymbol{x}, b<f<a\right)\right) \\
& =\log \left(\sqrt{2 \pi e \sigma^{2}} Z\right)+ \\
& \frac{1}{2 Z}\left[(\alpha-\mu) \mathcal{N}\left(\mu, \sigma^{2}\right)-(\beta-\mu) \mathcal{N}\left(\mu \mid \beta, \sigma^{2}\right)\right]
\end{aligned}
$$

where $Z=\Phi\left[\frac{\beta-\mu(\boldsymbol{x})}{\sigma}\right]-\Phi\left[\frac{\alpha-\mu(\boldsymbol{x})}{\sigma}\right], \Phi$ denotes the cumulative distribution function of standard normal distribution $\mathcal{N}$. The synthetic validation of this acquisition function can be found in [6].

In the proposed active learning framework, the sample $\overline{\boldsymbol{x}}$ which maximize the value of the acquisition function will be 


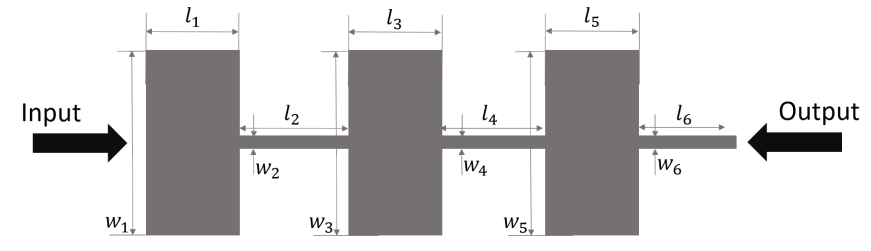

Fig. 2: Geometry of the low pass filter under study.

chosen as next sample point to be evaluated via expensive simulations (see Fig. 1). In order to estimate $\overline{\boldsymbol{x}}$, the following procedure is adopted: first the acquisition function is evaluated at 1000 candidate points chosen via Monte Carlo sampling, then the best candidate point is chosen as the starting point of an L-BFGS-B gradient based optimizer. This optimization process can be performed very efficiently, since the acquisition function $\alpha(\boldsymbol{x})$ depends on the GP model, which is very cheap to evaluate, and the training data $D_{n}$, which has already been computed.

\section{Target region parameterization}

In general, since the target region can have an arbitrary shape, its boundary can be intractable. Hence, a method to post-process the model, i.e. to parameterize the target region, is needed to provide continuous accessibility for designers. As shown in Algorithm 1, we approximate the target region by rejection sampling using the GP model, then we find a minimum volume hypercube to bound the resulting points. In particular, PCA is employed to find the principle axes of these data and use them to form an hypercube bounding the target region. PCA is widely used as a dimension reduction technique as it is able to describe the linear correlated data in an uncorrelated way under the principle axes. Here we demonstrate that it can provide a small bounding box. Besides, PCA offers an important advantage: it linearly transform points in the bounding box space to the original space, so the relation among the design parameters and the feasible region is clearly expressed. The bounding box search algorithm is implemented with scikit-learn [15].

\section{Application: Low PAss Filter Design}

In this Section, we adopt the proposed method to assist the design of a two port low pass stepped impedance microstrip filter [16], which layout is shown in Fig. 2. The filter is formed by six microstrips with different lenghts, while the following relation holds for the widths of the conductors [16]: $w_{1}=$ $w_{3}=w_{5}=w_{1,3,5}$ and $w_{2}=w_{4}=w_{6}=w_{2,4,6}$.

In particular, let us assume that a filter with a $3 \mathrm{~dB}$ cut-off frequency $f_{c}$ at $2.4 \mathrm{GHz}$ is desired, and that the relative permittivity $(\epsilon)$ and thickness $(h)$ of the substrate, and the width $w_{1,3,5}$ of the conductors are chosen as design parameters, varying in the range $\epsilon \in[2.1,6.3], h \in[1.2,3.2]$ $\mathrm{mm}, w_{1,3,5} \in[5.6,16.9] \mathrm{mm}$. The value of the remaining geometrical parameters of the filter is shown in Table I.

These choices lead to a large design space, including different substrate materials (i.e. FR4 $\left.(\epsilon=4.6), \mathrm{SiO}_{2}(\epsilon=3.9)\right)$
TABLE I: Low pass filter geometrical parameters

\begin{tabular}{|c|c|}
\hline Name & Geometry parameter \\
\hline microstrip lengths & $l_{1}=2.05 \mathrm{~mm}, l_{2}=6.63 \mathrm{~mm}, l_{3}=7.69 \mathrm{~mm}$, \\
& $l_{4}=9.04 \mathrm{~mm}, l_{5}=5.63 \mathrm{~mm}, l_{6}=2.41 \mathrm{~mm}$ \\
\hline microstrip widths & $w_{2}=w_{4}=w_{6}=0.428 \mathrm{~mm}$ \\
\hline
\end{tabular}

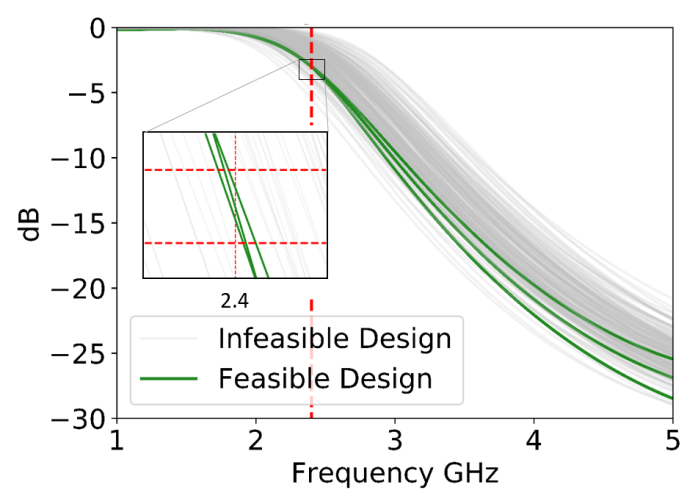

Fig. 3: Effect of the design parameters $\left(\epsilon, h, w_{1,3,5}\right)$ on the filter performance: the target region, defined as $\left|S_{12}\left(f_{c}\right)\right| \in$ $[-3.05 ;-2.95] \mathrm{dB}$, is shown by the red dashed line in the zoomed figure. While 200 designs are shown, only 3 fall within the target region.

and area required to build the filter. An example of the influence of the design parameters on the filter response is given in Fig. 3, where the element $\left|S_{12}\right|$ is computed for 200 different $\left(\epsilon, h, w_{1,3,5}\right)$ samples. It is interesting to remark that only $3\left(\epsilon, h, w_{1,3,5}\right)$ samples lead to feasible designs. In this example, the filter scattering parameters are computed using the quasi-analytical model described in [17] and implemented in MATLAB ${ }^{1}$.

Now, the feasible region identification problem can be formulated as follows:

$$
\begin{aligned}
& \text { Find }|| S_{12}\left(f_{c}, \epsilon, h, w_{1,3,5}\right)|\mathrm{dB}+3 \mathrm{~dB}| \leq \tau \\
& \text { subject to } \\
& \epsilon \in[2.1,6.3], h \in[1.2,3.2] \mathrm{mm}, w_{1,3,5} \in[5.6,16.9] \mathrm{mm}
\end{aligned}
$$

where $\tau$ is a suitable tolerance, chosen equal to $0.05 \mathrm{~dB}$. In order to compute the GP model, 15 initial $\left(\epsilon, h, w_{1,3,5}\right)$ samples are generated by Latin Hypercube Design (LHD) [18]. Then, additional 65 sampling points are sequentially evaluated, by maximizing the acquisition function to search for the target region.

The feasible region identified is shown in Fig. 4. In order to validate these results, $50000\left(\epsilon, h, w_{1,3,5}\right)$ samples are computed via Monte Carlo sampling. The predictions of the proposed active learning framework for these samples is compared with the results obtained by computing directly

\footnotetext{
${ }^{1}$ The Mathworks Inc., Natick, MA, USA
} 


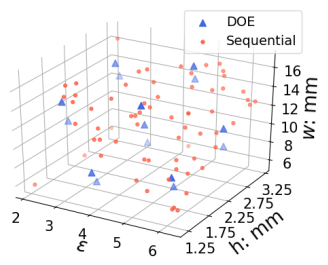

(a) Active learning sampling data view 1

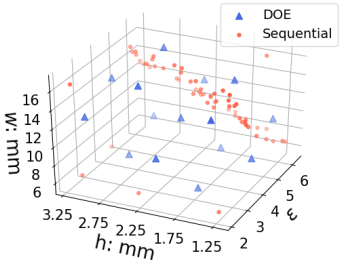

(b) Active learning sampling data view 2

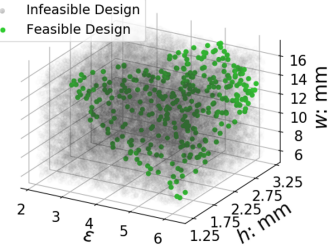

(c) Learned GP predict design data view 1

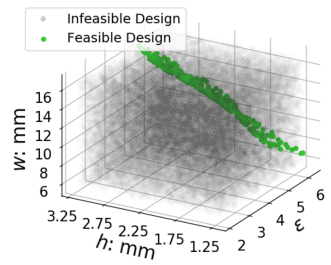

(d) Learned GP predict design data view 2

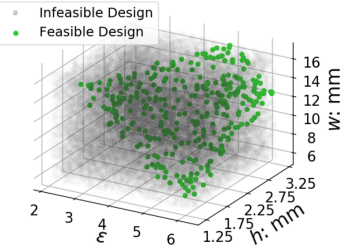

(e) Real design data view 1

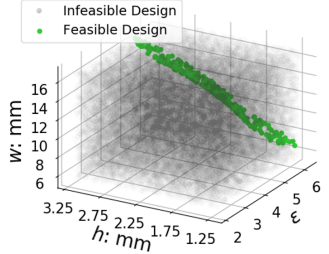

(f) Real design data view 2
Fig. 4: The feasible region estimated via active learning is compared with the one computed via direct simulation of the scattering parameters.

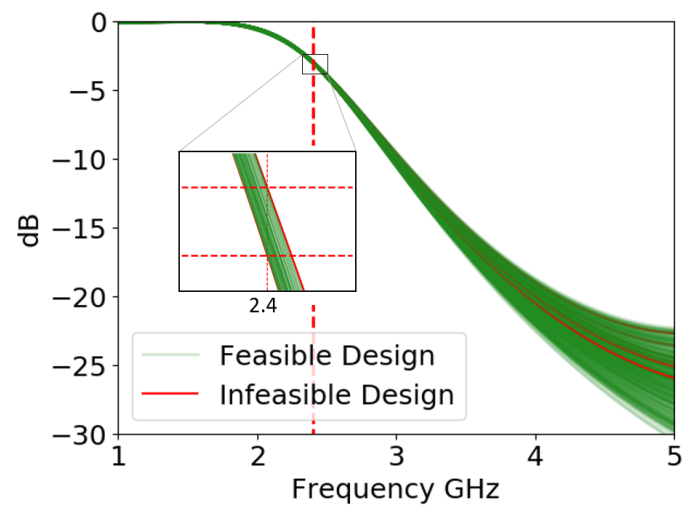

Fig. 5: Prediction curves in the target region, the false positive predicted infeasible design curves are near the target region.

the scattering parameters: it can be seen in Fig. 4 that the target region for this problem is similar to a ramp surface where the sequential sampling technique mostly focuses at (Fig. 4(a,b)), and that the data pattern predicted by the learned GP model shows high accuracy with respect to the scattering parameters simulations. To further demonstrate the accuracy of

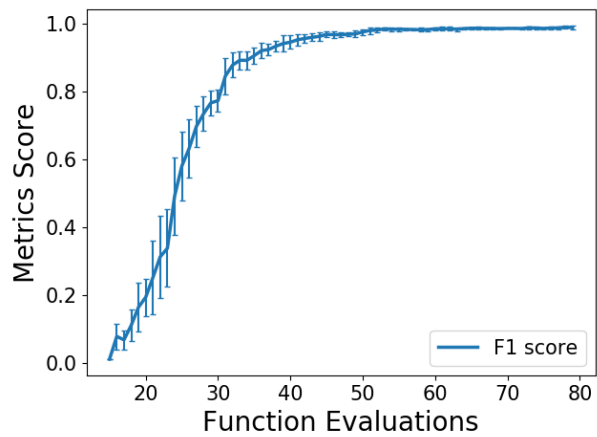

Fig. 6: Active learning progress plot.
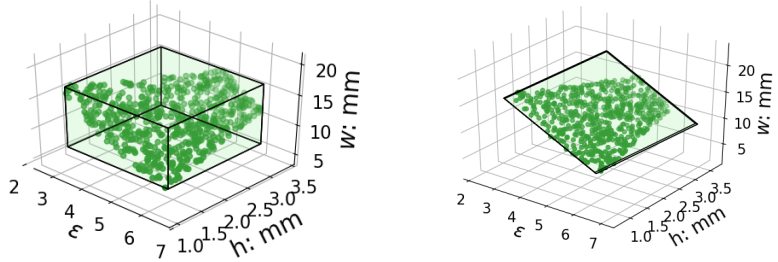

Fig. 7: The bounding target region generated by (a) an hypercube, (b) the proposed PCA-based approach.

the proposed method, the 323 predicted feasible designs in Fig. $4(\mathrm{c}, \mathrm{d})$ are simulated in the frequency range $[1 \mathrm{KHz} ; 5 \mathrm{GHz}]$ and the results are shown in Fig. 5. A high prediction accuracy can be observed: most of the curves are in the bounded region, where 6 false positives predicted by the GP model are also near the target region.

Finally, in order to test the robustness of the proposed methodology, the active learning process is repeated five times by using each time a different set of initial $\left(\epsilon, h, w_{1,3,5}\right)$ samples to compute the model. The GP model's accuracy is measured by the F1 score [6], which is often used to measure model's prediction accuracy. The F1 score is computed for each iteration by using the 50000 test data in Fig. 4 (e,f), and the corresponding progress plot is shown in Fig. 6. It can be seen that an accurate target region classification model is obtained after 50 iterations.

Once the feasible region is individuated, it is important to express it with respect to the design parameters $\left(\epsilon, h, w_{1,3,5}\right)$. A simple solution is to compute an hypercube encompassing the feasible region. However, this can lead to a gross overestimation of the feasible region, as shown in Fig. 7(a). A more accurate solution is to adopt the PCA-based approach described in Section II-D. Figure 7(b) illustrates the target region bounded by the PCA, showing a large reduction of the design space: more than $85 \%$ of the design space can be discarded and a much more informative target region can be provided to the designer for remainder of the design process. Indeed, based on the results of the proposed active learning framework, it is possible to design a filter satisfying the 
design specifications using materials with different relative permittivity for the substrate, and several combinations of $\left(h, w_{1,3,5}\right)$ can be chosen accordingly. Rather than providing a single design configuration (like in an optimization setting), the proposed technique allows designer to choose among several design solutions. This choice can be performed based on suitable criteria, such as overall cost or available area for the design.

In this example, the scattering parameters simulations are conducted with MATLAB $2018 a$ on a computer with Intel $(R)$ Core $(T M)$ i7-8650u @ $1.9 \mathrm{GHz}$ processor and 16 GB RAM. The active learning framework is implemented within GPFlowOpt [19].

\section{CONCLUSION}

In this paper, a Bayesian active learning method for electromagnetic design problems is presented. It is shown that the proposed technique is able to efficiently and accurately identify the regions of the design space that are of interest for the problem under study. Additionally, a PCA-based bounding box is adopted to clearly express the identified regions with respect to the design parameters. The proposed strategy is validated by means of a suitable application example.

\section{ACKNOWLEDGMENT}

The research is supported by Chinese Scholarship Council (grant number: 201906290032).

\section{REFERENCES}

[1] D. Spina, F. Ferranti, G. Antonini, T. Dhaene, L. Knockaert, and D. Vande Ginste, "Time-domain Green's function-based parametric sensitivity analysis of multiconductor transmission lines", IEEE Transactions on Components, Packaging and Manufacturing Technology, 2(9): 1510 1517,2012

[2] S. Grivet-Talocia, R. Trinchero, "Behavioral, parameterized, and broadband modeling of wired interconnects with internal discontinuities", IEEE Transactions on Electromagnetic Compatibility, 60(1): 77-85, 2017.

[3] B. Nouri, M. Nakhla, R. Achar, "Efficient simulation of nonlinear transmission lines via model-order reduction", IEEE Transactions on Microwave Theory and Techniques, 65(3): 673-683, 2016.

[4] I.T. Jolliffe, "Principal component analysis", 2nd ed., Springer-Verlag Inc., New York, 2002.

[5] J. Chen, L. Kang, G. Lin, "Gaussian process assisted active learning of physical laws", arXiv preprint arXiv:1910.03120, 2019.

[6] N. Knudde, I. Couckuyt, T. Dhaene, K. Shintani, "Active learning for feasible region discovery", accepted at 2019 International Conference on Machine Learning and Applications (ICMLA), Boca Raton, Florida, USA, Dec. 16-19, 2019.

[7] J. Miguel, H. Lobato, M. W.Hoffman, Z. Ghahramani, "Predictive entropy search for efficient global optimization of black-box functions", Advances in neural information processing systems, pag. 918-926, 2014.

[8] Z. Wang and S. Jegelka, "Max-value entropy search for efficient Bayesian optimization", Proceedings of the International Conference on Machine Learning (ICML), Sydney, Australia, Aug. 6 - 11, 2017.

[9] A. Kaintura, K. Foss, I. Couckuyt, T. Dhaene, O. Zografos, A. Vaysset, and B. Sorée, "Machine learning for fast characterization of magnetic logic devices", Proceedings IEEE Electrical Design of Advanced Packaging and Systems Symposium (EDAPS), Chandigarh, India, Dec. 16-18, 2018.

[10] J. Qing, Y. Hu, Y. Wang, Z. Liu, X. Fu, and W. Liu, "Kriging assisted integrated rotor-duct optimization for ducted fan in hover", Proceeding of AIAA Scitech 2019 Forum, San Diego, California, Jan. 7-11, 2019.
[11] G. Tapia, S. Khairallah, M. Matthews, W. E. King, A. Elwany, "Gaussian process-based surrogate modeling framework for process planning in laser powder-bed fusion additive manufacturing of 3161 stainless steel", The International Journal of Advanced Manufacturing Technology, 94(9-12): 3591-3603, 2018.

[12] B. Shahriari, K. Swersky, Z. Wang, R. P. Adams, and N. De Freitas, Taking the human out of the loop: A review of Bayesian optimization, Proceedings of the IEEE, 104(1): 148-175, 2015.

[13] C. E. Rasmussen, "Gaussian processes in machine learning", Summer School on Machine Learning, Springer, pag. 63-71, 2003.

[14] N. Houlsby, F. Huszar, Z. Ghahramani, J. M. Hernández-Lobato, "Collaborative gaussian processes for preference learning", Advances in neural information processing systems, pag. 2096-2104, 2012.

[15] F. Pedregosa, G. Varoquaux, A. Gramfort, V. Michel, B. Thirion, O. Grisel, M. Blondel, P. Prettenhofer, R. Weiss, V. Dubourg, J. Vanderplas, A. Passos, D. Cournapeau, M. Brucher, M. Perrot, and E. Duchesnay, "Scikit-learn: Machine learning in Python", Journal of Machine Learning Research, 12: 2825-2830, 2011.

[16] D. Spina, T. Dhaene, L. Knockaert, G. Antonini, "Polynomial chaosbased macromodeling of general linear multiport systems for time-domain analysis", IEEE Transactions on Microwave Theory and Techniques, 65(5): 1422-1433, May 2017.

[17] K. C. Gupta, R. Garg, I. Bahl, P. Bhartia, "Microstrip lines and slotlines", 2nd ed., Artech House Inc., Norwood, MA, 1996.

[18] F. Viana, G. Venter, and V. Balabanov, "An algorithm for fast optimal latin hypercube design of experiments", International journal for numerical methods in engineering, 82(2): 135-156, 2010.

[19] N. Knudde, J. Herten, T. Dhaene, I. Couckuyt, "Gpflowopt: A Bayesian optimization library using tensorflow", arXiv preprint arXiv:1711.03845, 2017. 